\title{
Massive hemorrhage caused by failure of elastic band release during endoscopic variceal ligation
}

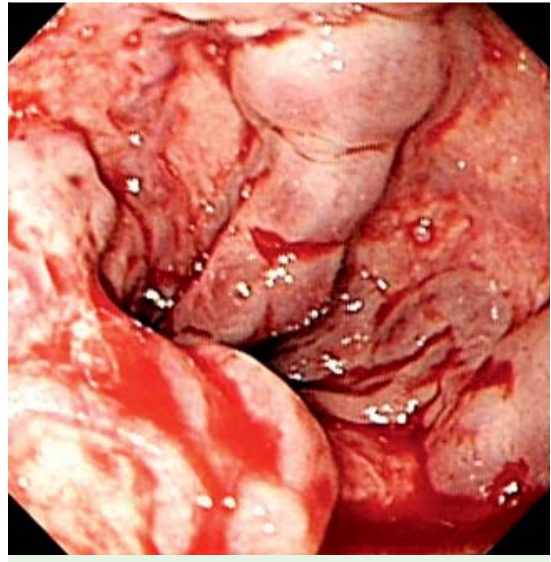

Fig. 1 Gastroscopy revealed severe esophageal varices with string-of-beads appearance in a 51-year-old man suffering from hematemesis and with a medical history of alcoholic cirrhosis. Active bleeding can be observed from the surface of the varicose veins.

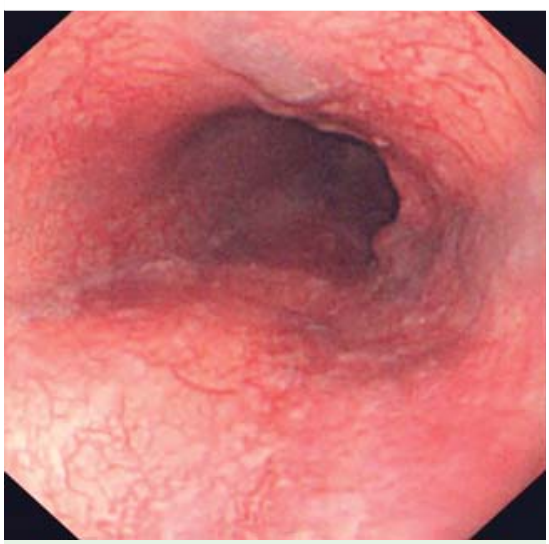

Fig. 3 Gastroscopy performed 14 months later showed the absence of severe esophageal varices and a well healed esophageal mucosa.

A 51-year-old man was transferred to our department as an emergency suffering from hematemesis for about half an hour. He had a medical history of alcoholic cirrhosis and had undergone splenectomy with portal azygos disconnection 6 years earlier. Emergency gastroscopy revealed severe esophageal varices with string-ofbeads appearance. Active bleeding could be observed from the surface of the varicose veins ( Fig. 1). Endoscopic variceal ligation was therefore indicated as the most appropriate therapeutic approach and a multiple band ligator (SpeedBand SuperView Super 7 Multiple Band Ligator; Boston Scientific, Natick, Massachusetts,
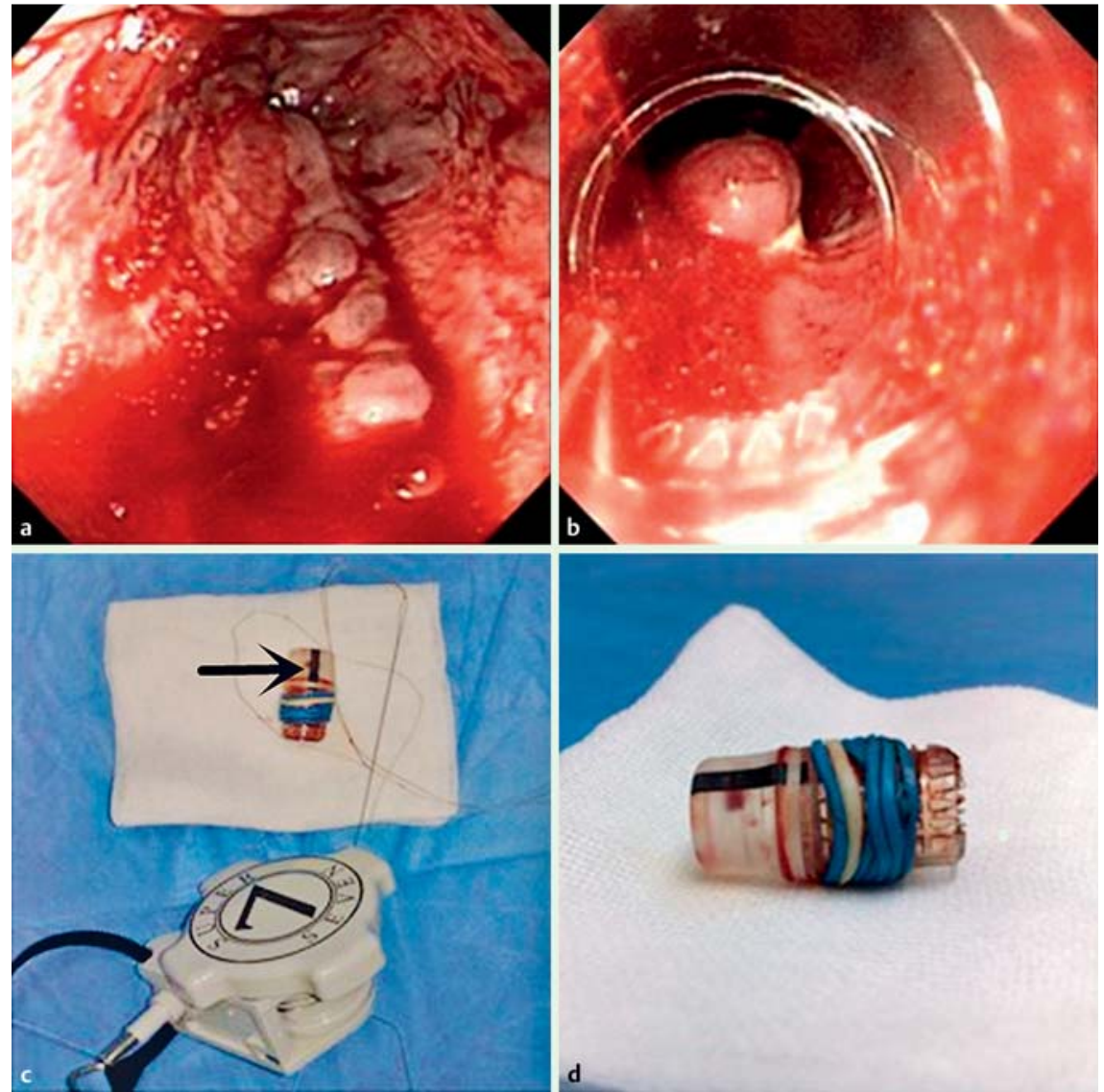

Fig. 2 a Massive hemorrhage from the broken varices caused by the powerful suction of the ligator without subsequent release of the elastic band. $\mathbf{b}$ The elastic band was successfully released after a new multiple band ligator had been installed and the bleeding subsequently stopped. $\mathbf{c}$ When checked carefully, the first multiple band ligator was found to have been installed incorrectly and the black marker line (arrow) was not aligned correctly with the biopsy channel of the endoscope. $\mathbf{d}$ This resulted in failure of elastic band release and the bands became entwined with each other.

United States) was used. When the esophageal varices and surrounding tissue were suctioned into the ligator cup at full power, the elastic band could not be released, leading to massive hemorrhage from the broken vessel as a result of the powerful suction ( Fig. 2a). Many attempts were made to release the elastic band but all failed. Therefore, we decided to withdraw gastroscopy and install a new multiple band ligator. The elastic band was successfully released this time and stopped the bleeding ( $\bullet$ Fig. 2 b).

The previous multiple band ligator was initially thought to be of substandard quality but was subsequently found to have been installed incorrectly and the black marker line was not aligned correctly with the biopsy channel of the endoscope ( $\bullet$ Fig.2c), leading to failure of elastic band release and ligation ( $\bullet$ Fig. 2 d).

The patient's hematemesis had stopped soon after the procedure was completed with the new multiple band ligator. An additional gastroscopy performed 14 months later confirmed the absence of severe esophageal varices ( $\bullet$ Fig.3) and the patient reported no further hematemesis or melena up to that time.

Endoscopic band ligation is an effective and safe method for the management of gastroesophageal varices and variceal hemorrhage in cirrhosis [1]. It is also considered to be safe and applicable beyond the prevention and management of gastroesophageal varices [2-5]; however 
few studies have reported complications or potential risks during its use. To our knowledge, this is the first report of endoscopic band ligation-related iatrogenic hemorrhage in the treatment of acute esophageal variceal bleeding, which was eventually well managed. In our experience, the multiple band ligator should be carefully checked and tested to confirm its normal function before use.

In conclusion, endoscopic band ligation is a relatively safe and useful method for the management of gastroesophageal varices if care is taken to ensure gentle and correct suction to avoid damage to fragile vessels or hemorrhage.

Endoscopy_UCTN_Code_CPL_1AH_2AC

Competing interests: None
Side Liu ${ }^{1, ~ *, ~ Y u e ~ L i ~}{ }^{1, ~ *}$, Chunli Cao ${ }^{1,2, ~ *, ~}$ Zelong Han ${ }^{1}$, Feng Xiong ${ }^{1}$, Wen $X u^{1}$, Xianfei Wang ${ }^{1}$

${ }^{1}$ Department of Gastroenterology, Nanfang Hospital, Southern Medical University, Guangzhou, China

2 Department of Gastroenterology, First Affiliated Hospital of Inner Mongolia Medical University, Huhhot, China

\section{Acknowledgments \\ $\nabla$}

The project was supported by Guangdong Province Universities and Colleges Pearl River Scholar Funded Scheme.

\section{References}

1 Garcia-Tsao G, Sanyal AJ, Grace ND et al. Prevention and management of gastroesophageal varices and variceal hemorrhage in cirrhosis. Am J Gastroenterol 2007; 102: 2086-2102

2 Han JH, Lee TH, Jung Y et al. Rescue endoscopic band ligation of iatrogenic gastric perforations following failed endoclip closure. World J Gastroenterol 2013; 19: 955 959

3 Li Y, Han Z, Zhang $W$ et al. Successful closure of lateral duodenal perforation by endo-

* These authors contributed equally to this study. scopic band ligation after endoscopic clipping failure. Am J Gastroenterol 2014; 109: 293-295

4 Nan G, Siyu S, Shiwei S et al. Hemoclip-reinforced and EUS-assisted band ligation as an effective and safe technique to treat small GISTs in the gastric fundus. Am J Gastroenterol 2011; 106: 1560-1561

5 Ji JS, Cho YS. Endoscopic band ligation: beyond prevention and management of gastroesophageal varices. World J Gastroenterol 2013; 19: $4271-4276$

Bibliography

Dol http://dx.doi.org/

10.1055/s-0034-1390862

Endoscopy 2015; 47: E28-E29

(c) Georg Thieme Verlag KG

Stuttgart · New York

ISSN 0013-726X

\section{Corresponding author}

\section{Side Liu, MD}

Guangdong Provincial Key Laboratory

of Gastroenterology

Department of Gastroenterology

Nanfang Hospital

Southern Medical University

Guangzhou 510515

China

Fax: +86-20-87280770

liuside@163.com 